\title{
Simulação e Otimização de Sistemas de Sinalização Semafórica
}

\author{
Alternative Title: Simulation and Optimization of Traffic Light Systems \\ M.B. Pereira \\ Departamento de Ciência da Computação - \\ UFLA \\ Caixa Postal 3037 - 37200-000 \\ Lavras - MG - Brasil \\ matheusbarros@computacao.ufla.br \\ D.L. Pereira \\ Departamento de Ciência da Computação - \\ UFLA \\ Caixa Postal $3037-37200-000$ \\ Lavras - MG - Brasil \\ dilson.pereira@dcc.ufla.br
}

\begin{abstract}
RESUMO
O presente artigo propõe um simulador de trânsito, capaz de reproduzir o comportamento de um sistema real sob determinadas condições. Em conjunto ao simulador, é proposto também um otimizador de tempos semafóricos. Desta forma, têm-se como objetivos principais: simular um sistema de acordo com um conjunto de parâmetros de entrada e retornar ao usuário os resultados obtidos, como o tempo médio de espera de cada carro nos semáforos, ou descobrir qual a melhor configuração dos sinais de trânsito em cada cruzamento, buscando atingir menores tempos de espera. O modelo de simulação tem base no paradigma de simulação orientada a eventos discretos e se baseia na ideia de que é possível determinar o tempo de espera de um veículo no semáforo com base no estado do semáforo no momento da chegada deste veículo e no momento de saída do veículo anterior. O otimizador tem como base a metaheurística algoritmo genético. O simulador de cruzamentos foi testado e seus dados comparados com informações obtidas em um sistema real, resultando em valores que ficaram bem próximos aos reais. Além disso, os resultados obtidos com o otimizador indicam que a ferramenta pode ser útil para o auxílio na tomada de decisões e é capaz de gerar sugestões de melhorias nas configurações semafóricas.
\end{abstract}

\section{Palavras-Chave}

simulação, otimização, sinalização semafórica, algoritmos genéticos

\footnotetext{
ABSTRACT

This paper aims at developing an algorithm for simulating traffic systems and an algorithm for optimizing traffic light times. The simulation algorithm makes use of a simulation model based on the discrete event simulation paradigm. Given a set of input parameters, the simulation algorithm pro-

Permission to make digital or hard copies of all or part of this work for personal or classroom use is granted without fee provided that copies are not made or distributed for profit or commercial advantage and that copies bear this notice and the full citation on the first page. To copy otherwise, or republish, to post on servers or to redistribute to lists, requires prior specific permission and/or a fee.

SBSI 2017 June $5^{t h}-8^{t h}, 2017$, Lavras, Minas Gerais, Brazil

Copyright SBC 2017.
}

duces data such as the average vehicles' wait times at traffic lights. The simulation model assumes that it is possible to determine a vehicle's wait time at a traffic light if the light's state upon the vehicle's arrival and the moment the previous vehicle left the light are known. The optimization algorithm is based on the Genetic Algorithm metaheuristic, its goal is to find the best configuration of the traffic lights in the network, aiming at obtaining the least wait times. The algorithms were tested on real and artificial instances. The obtained results indicate that the algorithms proposed here can be useful tools to aid in decision making.

\section{CCS Concepts}

-Mathematics of computing $\rightarrow$ Mathematical optimization; •Computing methodologies $\rightarrow$ Model verification and validation; Discrete-event simulation;

\section{Keywords}

simulation, optimization, traffic light systems, genetic algorithms

\section{INTRODUÇÃO}

O número cada vez maior de veículos vem sobrecarregando a infraestrutura de trânsito nas cidades. Um bom planejamento do controle semafórico é essencial para evitar diversos problemas de tráfego, como fila excessiva de veículos para transpor uma interseção e ocorrência de acidentes [4].

Ferramentas de simulação e otimização vêm sendo empregadas com sucesso na análise de sistemas de tráfego, como o TRANSYT (Traffic Network Study Tool), criado pelo Dr. Dennis I. Robertson, no RRL - Road Research Laboratory (hoje TRL - Transport Research Laboratory). O problema, entretanto, está no fato de muitos desses simuladores serem inacessíveis ou pagos. Além disso, simuladores estrangeiros podem não se adequar bem às particularidades do trânsito brasileiro, como, por exemplo, tempos de verde de segurança configurados por grupo semafórico e não por estágio, casos de ciclos duplos assimétricos e botoeiras de pedestres chamando estágios específicos em cruzamentos. Esforços para criação de simuladores em âmbito nacional são raros. Exceto, por exemplo, o SIRI (Simulador de Redes de Semáforos), software desenvolvido e utilizado pela CET/SP, mas que não é acessível externamente à companhia [10]. 
De acordo com Hillier e Lieberman (2006), simulação envolve o uso de um computador para imitar (simular) a operação de todo um processo ou sistema. É frequentemente usada, por exemplo, para analisar sistemas estocásticos que continuarão a operar indefinidamente [8]. No presente caso, trata-se de uma simulação orientada a eventos discretos. Leemis e Park (2006) definem um modelo de simulação orientada a eventos discretos por meio de três atributos: estocástico (algumas variáveis do sistema são aleatórias), dinâmico (a evolução no tempo das variáveis do sistema é importante) e discreto (mudanças significativas nas variáveis do sistema estão associadas a eventos que ocorrem em instantes discretos de tempo). Já otimização é uma área de estudo que se preocupa em encontrar a melhor configuração para sistemas $[9]$.

A simulação proporciona uma maneira mais cômoda de conhecer melhor o sistema, prever seu funcionamento sob determinadas circunstâncias, propor ou verificar o efeito de possíveis mudanças. O uso de técnicas de simulação e otimização pode proporcionar economia de tempo, dinheiro e outros recursos importantes.

Segundo o Manual Brasileiro de Sinalização de Trânsito Volume V, a estratégia de controle em rede permite a programação da sinalização semafórica visando não somente o desempenho do tráfego em cada interseção mas, sobretudo, o seu desempenho global ao longo do conjunto de cruzamentos [4]. Pode-se subdividir tal estratégia em, basicamente, dois tipos de operação. Na operação em tempo fixo, os tempos semafóricos não mudam durante um determinado período e são calculados para atender a volumes médios de tráfego nas aproximações, definindo um plano semafórico. Os sistemas atuados, ou sistemas que variam conforme a demanda veicular, são usados nos casos em que o tráfego não apresenta um comportamento cíclico, ocorrendo variações de volume veicular de um dia para o outro [10]. A abordagem de tempo fixo foi a escolhida para ser tratada na simulação por ser o tipo de caso mais comum, principalmente em pequenas e médias cidades, podendo, assim, ser útil para a maior parte das cidades brasileiras.

Além do TRANSYT e SIRI citados anteriormente, outros algoritmos e modelos já foram propostos, tanto para controle de tempos fixos quanto para controles adaptativos em tempo real. Entre eles, encontra-se o ATEFI, cujo modelo de otimização calcula o índice de desempenho da rede, mas diferencia-se por determinar o tempo de verde e a defasagem ótima através da heurística Hill Climbing [3]. Para controles em tempo real, pode-se citar [1], [2] e [6]. Além disso, [11] faz uma revisão das diversas estratégias de controle de tráfego existentes.

O presente trabalho tem como objetivo propor um simulador orientado a eventos discretos e um algoritmo de otimização baseado no algoritmo genético para sistemas de sinalização semafórica. Em linhas gerais, dado um conjunto de cruzamentos com determinadas características e pontos de origem de tráfego, busca-se descobrir uma boa configuração semafórica que diminua o tempo média de espera dos veículos ao longo da rede.

\section{SIMULAÇÃO}

Nesta seção, será proposto um modelo de simulação, isto é, uma abstração do sistema a ser simulado e seu funcionamento. O modelo de simulação proposto segue o tipo de controle semafórico de tempo fixo. Segundo o Manual Bra- sileiro De Sinalização De Trânsito Volume V, este tipo de controle apresenta como uma de suas principais vantagens o fato de seu custo de implantação ser inferior ao de controle atuado, que permite alteração nas configurações semafóricas em tempo real. Entretanto, o controle de tempo fixo é pouco eficiente para locais, ou períodos do dia, em que o volume de tráfego sofra muitas variações. De qualquer modo, este é o modelo implementado na maioria das cidades brasileiras [4].

Como passo inicial para o entendimento do modelo de simulação de redes de tráfego proposto, será discutido na próxima subseção um caso mais simples, a simulação de vias únicas em cruzamentos sinalizados.

\subsection{Simulação de uma única via em um cru- zamento sinalizado}

Um primeiro passo em um estudo de simulação é a definição de um modelo de simulação, uma abstração do sistema e suas entidades. Para o caso da simulação semafórica de uma única via em um cruzamento sinalizado, tais entidades são as próprias vias, faixas, veículos e semáforos, discutidas a seguir.

\subsubsection{Vias de entrada}

Vias de entrada são o conjunto de faixas de trânsito de uma mesma rua que levam os veículos em direção ao cruzamento, ou seja, são as fontes de entrada de veículos. Além das faixas, as vias de entrada são compostas de um semáforo e possuem como dado de entrada necessário o tempo médio de chegada entre veículos. Este parâmetro indica uma média de tempo em segundos para um veículo chegar no cruzamento pela via de entrada após a chegada do último veículo. Como dado de saída, deseja-se gerar o tempo de espera total de veículos e o número de veículos que passaram pela via ao longo da simulação, o que permite calcular o tempo médio de espera.

\subsubsection{Faixas}

Vias de entrada são subdivididas em faixas. Cada faixa possui um conjunto de direções que podem-se seguir ao atravessar o cruzamento, ou seja, um conjunto de vias de saída de destino. É possível que faixas distintas de uma mesma via de entrada apresentem direções diferentes.

\subsubsection{Semáforos}

Os cruzamentos sinalizados possuem semáforos que coordenam a passagem dos veículos. Cada semáforo $s$ possui um tempo de sinal aberto (ou verde), denotado $T_{\text {verde }}(s)$, e um tempo de sinal fechado (ou vermelho), denotado $T_{\text {vermelho }}(s)$. O tempo de sinal amarelo será considerado parte do tempo verde, abordagem realizada de forma semelhante a [5].

\subsubsection{Veículos}

Veículo são as entidades que trafegam pelas vias e faixas, e que têm seu movimento coordenado pelos semáforos. De modo geral, são dados de entrada de veículos o tempo médio de reação à abertura do semáforo, $T_{\text {reage, }}$ e o tempo médio, $T_{\text {passa }}$, que um veículo leva para atravessar o semáforo, que depende do seu comprimento e velocidade ao arrancar. Em particular, para cada veículo $v$, tem-se o seu tempo de chegada na via, $T_{\text {chega }}(v)$, e, como saída, calcula-se o tempo de saída, $T_{s a i}(i)$, a partir dos quais é possível calcular o tempo de espera do veículo no semáforo, 
$T_{\text {espera }}(i)=T_{\text {sai }}(i)-T_{\text {chega }}(i)$.

O parâmetro $T_{\text {reage }}$ corresponde ao tempo levado para o primeiro veículo da fila poder alcançar o semáforo perante a mudança do sinal de vermelho para verde. Portanto, tal tempo considera desde os segundos levados para o veículo começar a acelerar até o tempo necessário para que ele se desloque até o semáforo para sair do cruzamento. Este tempo é considerado apenas para o primeiro veículo da fila no momento da abertura do sinal, pois assume-se que os demais veículos se aproximarão o máximo possível do veículo à sua frente, de modo a anular seu tempo de reação.

O parâmetro $T_{\text {passa }}$, indica quantos segundos são levados desde a saída do veículo anterior até a saída do atual, ou, quantos segundos o veículo leva para percorrer seu próprio comprimento ao atravessar o semáforo. Tal tempo pode ser um valor médio real conhecido para uma determinada via de entrada, que será utilizado para todos os casos, ou pode ser gerado aleatoriamente para cada veículo, seguindo um determinado parâmetro, como a proporção de ocorrências de veículos grandes e pequenos na via, por exemplo.

Uma maneira para se obterem os valores de $T_{\text {chega }}(v)$, para cada veículo $v$, seria recebê-los como entrada do sistema. No entanto, esta seria uma abordagem pouco prática. Desta forma, modelam-se estes valores como uma variável aleatória, a partir da qual os valores específicos são gerados pelo sistema. De acordo com Leemis e Park [9], o uso de uma variável aleatória uniforme, $U(a, b)$, em que todos valores em um intervalo $(a, b)$, para parâmetros reais $a$ e $b$, são igualmente possíveis, é inadequado. Isto ocorre pois, em situações reais, alguns valores são mais prováveis que outros. Em muitos casos de simulação a eventos discretos, como o presente, a simulação requer uma variável aleatória contínua, $X$, que pode assumir qualquer valor positivo, de maneira que valores menores de $X$ são mais prováveis do que maiores. Para gerar tal variável aleatória é necessário uma transformação não linear que mapeia valores da variável aleatória $U(0,1)$ para valores de $X$ entre 0 e $\infty$. Isto é feito "esticando" valores altos de $U$ mais do que menores. Uma forma comum de se fazer isso é atribuindo-se $X=-\mu \ln (1-U)$, em que $\mu>0$ e ln é o logaritmo natural. Esta transformação gera o que é conhecido como variável aleatória exponencial $\operatorname{Exp}(\mu)$ com média $\mu$. Para o caso do modelo proposto, $\mu$ é a média entre a chegada de veículos e $U$ é uma variável do tipo double gerada aleatoriamente pelo computador. Sendo assim, dado um veículo $v$, gera-se $U(v)$ em $U(0,1)$, a partir do qual gera-se $X(v)$ em $\operatorname{Exp}(\mu)$, e determina-se $T_{\text {chega }}(v)=T_{\text {chega }}(v-1)+X(v)$, caso $v>1$, e $T_{\text {chega }}(1)=X(v)[9]$.

Segundo Hillier et al. [8], a distribuição exponencial é simples e computacionalmente tratável, mas, ao mesmo tempo, realista, fornecendo boas previsões para modelos baseados em teoria das filas.

\subsubsection{Modelo de simulação para uma única via si- nalizada}

Assuma que, em um dado momento $t$, Sinal( $t$ ) representa o estado do semáforo, podendo assumir os valores "vermelho" ou "verde". Assuma também que $A(t)$ representa o momento da próxima abertura do semáforo após o momento $t$.

O seguinte modelo relaciona o momento de saída de um veículo $v$ com os dados de entrada do sistema e momentos de saída dos veículos anteriores.

- $\operatorname{Se} T_{\text {chega }}(v) \geq T_{\text {sai }}(v-1)+T_{\text {passa }}(v-1)$ e $S\left(T_{\text {chega }}(v)\right)=$ verde: $T_{\text {sai }}(v)=T_{\text {chega }}(v)$. Isto é, se quando um determinado veículo chegar ao semáforo o veículo anterior já tiver saído e o semáforo estiver verde, então seu tempo de saída é igual ao tempo de chegada.

- Se $T_{\text {chega }}(v) \geq T_{\text {sai }}(v-1)+T_{\text {passa }}(v-1)$ e $S\left(T_{\text {chega }}(v)\right)=$ vermelho: $T_{\text {sai }}(v)=T_{\text {chega }}(v)+A\left(T_{\text {chega }(v)}\right)+T_{\text {reage }}(v)$. Ou seja, se o veículo chegar a um semáforo vazio e fechado, deverá aguardar a próxima abertura.

- Se $T_{\text {chega }}(v)<T_{\text {sai }}(v-1)+T_{\text {passa }}$ e $S\left(T_{\text {sai }}(v-1)+\right.$ $\left.T_{\text {passa }}(v-1)\right)=$ verde então $T_{\text {sai }}(v)=T_{\text {sai }}(v-1)+$ $T_{\text {passa }}(v-1)$. Este caso indica que se, no momento em que um veículo $v$ chegar ao semáforo o veículo anterior ainda não tiver saído completamente, mas, após sua saída, o semáforo estiver verde, então $v$ sai logo depois do veículo anterior.

- Se $T_{\text {chega }}(v)<T_{\text {sai }}(v-1)+T_{\text {passa }}(v-1)$ e $S\left(T_{\text {sai }}(v-\right.$ $\left.1)+T_{\text {passa }}(v-1)\right)=$ vermelho: $T_{\text {sai }}(v)=T_{\text {sai }}(v-1)+$ $T_{\text {passa }}(v-1)+A\left(T_{\text {sai }}(v-1)+T_{\text {passa }}(v-1)\right)+T_{\text {reage }}(v)$. Isto é, se no momento em que o veículo $v$ chegar ao semáforo o veículo anterior ainda não tiver saído, e quando este veículo sair o semáforo estiver vermelho, então $v$ precisará esperar a próxima abertura do sinal.

O modelo descrito acima é utilizado como base para a simulação de cruzamentos, discutida na próxima seção.

\subsection{Simulação de cruzamentos}

Cruzamentos são compostos de vias de entrada e vias de saída. Através das vias de entrada, os veículos se dirigem ao cruzamento. Através das vias de saída, os veículos saem do cruzamento. Com base nos tempos de espera de cada uma das vias de entrada, é possível calcular o tempo de espera total e médio do cruzamento como um todo.

A simulação de um cruzamento sinalizado é, então, feita simulando-se cada uma de suas vias de entrada separadamente, conforme o modelo descrito na Seção 2.1.5. Os veículos são gerados com base nos dados de entrada passados pelo usuário e a função exponencial descrita na Seção 2.1.4.

\subsubsection{Agrupamentos semafóricos}

Um aspecto que o modelo pode considerar é a possibilidade de realizar agrupamentos semafóricos em alguns cruzamentos sinalizados de acordo com a necessidade do usuário. O Manual Brasileiro de Sinalização semafórica define movimentos com origens diferentes cujas trajetórias se interceptam (que têm origem em aproximações diferentes e que se cruzam em algum ponto da área de conflito) ou convergem (que têm origem em diferentes aproximações e possuem mesmo destino) em algum ponto da área de conflito como conflitantes. O usuário poderá identificar os movimentos não conflitantes para poder gerar grupos semafóricos que, segundo o manual, são conjuntos de semáforos com indicações luminosas idênticas que controlam grupos de movimentos que recebem simultaneamente o direito de passagem [4]. Quanto mais grupos semafóricos forem gerados, maior será o número de vias em movimento ao mesmo tempo, o que diminuirá o tempo de ciclo dos semáforos e fará os veículos terem que, em média, esperar menos em suas filas.

Para simular casos em que faixas de uma via podem fazer parte de diferentes grupos semafóricos, o usuário pode subdividir esta via em outras vias, de modo que todas as 
faixas de uma via pertençam ao mesmo grupo semafórico, tomando cuidado para que as médias de chegada de veículos em cada via sejam ajustadas adequadamente.

\subsection{Simulação de uma rede de cruzamentos}

Esta seção descreve como, com base nas ideias discutidas nas seções anteriores, é possível simular uma rede de tráfego. De posse de um simulador deste tipo, o operador pode simular setores de uma cidade, ou uma pequena cidade, por exemplo.

Uma rede de tráfego é composta por cruzamentos com ou sem sinalização conectados entre si. Uma ou mais vias de entrada devem ser selecionadas pelo usuário para serem fonte de veículos para a rede, sendo o processo de geração de veículos feito conforme a Seção 2.1.4. Da mesma forma, uma ou mais vias de saída devem ser selecionadas para consumirem os veículos que saem da rede, isto é, veículos que passarem por tal via serão removidos do sistema. Além disso, é necessário que os cruzamentos sejam conectados, de forma que a via de saída de um leve veículos para a via de entrada de outro. Tal conexão é uma nova entidade adicionada ao modelo e requer que seja informado o tempo de deslocamento médio de um veículo para se locomover pela via $e$ que conecta os cruzamentos, denotado $T_{\text {desloca }}(e)$. Na prática, este tempo pode ser medido utilizando-se a velocidade média dos veículos na via e a distância entre os cruzamentos.

Um novo parâmetro a ser considerado na simulação é o tempo de atraso para a primeira abertura de um semáforo em cada cruzamento sinalizado, sendo tal valor necessário para que seja determinado o estado inicial do sistema. É considerado que o usuário deverá adicionar as vias ou grupos semafóricos pela ordem de abertura desejada. O parâmetro determina, então, o momento após o tempo zero da simulação em que a primeira via adicionada abrirá seu semáforo. Um bom valor de atraso permite que sejam maiores as chances de um veículo encontrar o semáforo do próximo cruzamento aberto após sair de um outro. O valor mínimo para o atraso é zero e ele não deve ser maior que $T_{\text {vermelho }}$ da primeira via do cruzamento, o que indicaria um tempo que superaria o tempo de ciclo do semáforo.

O uso e necessidade do valor de atraso para abertura inicial é justificado pelo Manual Brasileiro de Sinalização Semafórica - Volume V, página 66, da seguinte forma:

A programação da sinalização semafórica operando em rede implica, além da determinação dos parâmetros necessários para a programação isolada, a definição de um parâmetro adicional, denominado defasagem. Esse parâmetro estabelece, tomando como base uma referência temporal especificada, o momento de iniciar o verde de um estágio para cada interseção que integra a rede.

É necessário que seja informada, também, para cada via de entrada $e$ em um cruzamento e para cada possível via de saída $e^{\prime}$ para $e$, a probabilidade de um veículo trafegando por e dirigir-se para $e^{\prime}$, denotada $D\left(e, e^{\prime}\right)$.

\subsubsection{Modelo de simulação de rede de cruzamentos}

Para simular as vias da rede, o algoritmo baseado no modelo da Seção 2.1.5 continua aplicável, o que muda é que, nas vias internas, ao invés de gerar os veículos conforme a distribuição exponencial, os veículos são fruto de outros cruzamentos, enquanto nas vias de origem, os veículos são gerados normalmente, conforme a distribuição exponencial.

Sendo assim, não é possível simular cada cruzamento ou via de forma isolada, como na simulação de cruzamentos isolados, da Seção 2.2, pois a saída de alguns é a entrada para outros. Para manter a sincronia entre os cruzamentos, utiliza-se uma fila de chegadas que envolve toda a rede. Cada chegada armazenada na fila possui o cruzamento e via de entrada à qual pertence, um veículo, o momento da chegada e a via de saída de destino, escolhida aleatoriamente dentre as possíveis vias de saída, conforme o parâmetro $D$, descrito acima. Inicialmente, as chegadas são geradas de forma aleatória para todas as vias de entrada que são fonte de veículos para a rede, conforme a função exponencial descrita na Seção 2.1.4.

A fila é ordenada de forma não decrescente pelos momentos das chegadas. A simulação, então, consiste em retirar o primeiro elemento da fila, determinando o veículo $v$ e sua via $e$ correspondentes, processá-los de acordo com o modelo descrito na Seção 2.1.5, determinar a via de destino $e^{\prime}$, conforme o parâmetro $D\left(e, e^{\prime}\right)$, o momento de chegada no cruzamento de $e^{\prime}$, conforme o tempo de deslocamento entre os cruzamentos das vias, $T_{\text {desloca }}\left(e^{\prime}\right)$, e inserir a nova chegada na fila. Entretanto se o veículo se dirigir à uma via de saída da rede, ele é removido do sistema, não sendo reinserido na fila. O processo se repete até a fila esvaziar-se.

\section{Processamento de uma chegada em uma via não sina- lizada.}

Os cruzamentos não sinalizados diferenciam-se justamente por não possuírem semáforos. Portanto, para cada via de entrada, é necessário saber qual sua prioridade em relação às outras vias. Isso é necessário pois a passagem não é dependente de um semáforo, e sim da aproximação de outros veículos que estão em vias com prioridade maior. Portanto, para decidir o momento de saída em cruzamentos não sinalizados é necessário verificar se em um determinado tempo de segurança para travessia não existe a aproximação de veículos em vias prioritárias. Tal tempo de segurança indica a quantos segundos do cruzamento um outro veículo, se aproximando por uma via prioritária, vai impedir a passagem do atual, sendo também um valor de entrada passado pelo usuário.

Um veículo que se dirige para uma via de saída com um movimento de maior prioridade sempre passará direto pelo cruzamento, ou seja, não possui tempo de espera. Entretanto se o movimento não for prioritário é necessário consultar as chegadas posteriores.

Uma abordagem mais realista seria realizar de forma paralela o processamento das chegadas posteriores à atual até ser possível confirmar que nenhuma outra aproximação a atrasará. Isto ocorre pois é possível que um veículo passe diretamente por um ou mais cruzamentos e chegue dentro do tempo de segurança para travessia da chegada atual, sendo que tal caso só é identificado após processar o veículo que passou pelo cruzamento adjacente. O problema, entretanto, é que se houver uma outra chegada em um cruzamento sem semáforo, outro processamento paralelo será iniciado novamente em cadeia e assim sucessivamente. Isto resultará em um consumo de recursos computacionais elevado e que poderá ser descartado após a tomada da decisão em relação a travessia do veículo, já que tal decisão altera a ordem da fila. 
Outra abordagem possível seria considerar que todas as chegadas em um cruzamento sem semáforo por padrão não são atrasadas, mas isto representaria um modelo muito distante da realidade, o que não seria adequado. Foi optado, então, por consultar a fila de chegadas em busca de uma aproximação com movimento prioritário à atual, mas sem realizar processamento paralelo. Assim, caso não haja uma chegada que possa atrasar a atual no tempo de segurança para travessia da via, ela será processada. Caso contrário, ela será atrasada. Apesar de não ser tão realista quanto a primeira abordagem, esta última é suficientemente próxima da realidade para não comprometer a simulação, já que o caso que ela não abrange possui baixas chances de acontecer.

Dessa forma, caso seja verificado que o veículo não poderá atravessar o cruzamento naquele momento, seu tempo de espera será incrementado assim como o de todos os outros que estão com ele na fila de sua via de entrada. As chegadas de todos estes veículos também serão atrasadas para que o primeiro tente atravessar novamente somente após a travessia do veículo que o atrasou.

\section{OTIMIZAÇÃO}

O algoritmo de otimização é aplicado em conjunto com os modelos descritos anteriormente. Seu objetivo principal é encontrar uma configuração semafórica para a rede de cruzamentos que apresente uma média de tempo de espera baixa para os veículos.

Após geradas as chegadas e os grupos semafóricos (caso existam), o algoritmo de otimização precisa descobrir uma boa configuração dos semáforos para diminuir o tempo médio de espera dos veículos. O método escolhido foi a utilização de um algoritmo genético para conseguir tal resultado.

\subsection{Algoritmo genético}

Algoritmos genéticos são uma meta-heurística. Segundo Hillier et al. [8], um método heurístico é um procedimento que provavelmente vai encontrar uma boa solução viável, mas não necessariamente uma solução ótima, para o problema específico em questão, não sendo possível dar uma garantia sobre a qualidade da solução obtida. Já uma metaheurística é um método de resolução geral que fornece tanto uma estrutura quanto diretrizes de estratégia gerais para desenvolver um método heurístico específico que se ajuste a um tipo de problema particular. Algoritmos genéticos fazem uma analogia à teoria da evolução biológica formulada por Charles Darwin em meados do século XIX. As soluções viáveis para determinado problema correspondem aos membros de dada espécie, na qual a adaptação de cada membro agora é medida pelo valor da função objetivo. Para cada iteração (geração) de um algoritmo genético, a população atual é formada pelo conjunto de soluções experimentais que estão sendo consideradas. Alguns membros da população sobrevivem, se tornam pais (formando pares aleatórios) que depois têm filhos (novas soluções experimentais) que compartilham algumas das características de ambos os pais. Já que os membros mais adaptados têm mais chance de se tornarem pais que outros, um algoritmo genético tende a gerar populações melhores de soluções experimentais a medida que prossegue. Ocasionalmente ocorrem mutações de modo que certos filhos também adquirem características que nenhum dos pais possui. Finalmente, a sobrevivência dos mais adaptados deve tender a levar um algoritmo genético a uma so- lução experimental que é, pelo menos, próxima da solução ótima [7].

A seguir, são apresentados os principais componentes de um algoritmo genético desenvolvido para a otimização dos tempos dos semáforos em um único cruzamento.

\subsection{Algoritmo genético para cruzamentos si- nalizados isolados}

\subsubsection{Representação dos indivíduos}

Os indivíduos do algoritmo são representados por um vetor em que cada posição é o tempo de sinal verde de um respectivo grupo semafórico do cruzamento. Simula-se o cruzamento naquela determinada condição e obtêm-se os tempos médios de espera de cada uma das vias.

\subsubsection{Função objetivo}

O objetivo do algoritmo é minimizar o maior tempo de espera entre todas as vias do cruzamento.

\subsubsection{População inicial}

Inicialmente, são gerados 100 indivíduos aleatórios, ou seja, são geradas 100 valores aleatórios de tempos de sinal aberto para cada grupo semafórico. Isto é feito seguindo a recomendação do Manual Brasileiro de Sinalização de Trânsito de não permitir ciclos com mais de 120 segundos. Assim, o somatório do tempo aberto de cada grupo semafórico deve ser menor que 120 .

\subsubsection{Seleção de indivíduos}

Para a seleção dos indivíduos que se cruzarão para gerar novos indivíduos, utiliza-se um torneio. Dois indivíduos distintos aleatórios são selecionados e comparados. O que possuir o menor maior tempo de espera para uma via é escolhido. Em seguida dois outros indivíduos aleatórios são escolhidos e o melhor entre eles é selecionado. Ambos os vencedores se cruzam para formar o novo indivíduo da próxima geração. Ao todo, serão gerados 200 pares aleatórios.

\subsubsection{Cruzamento de indivíduos}

O cruzamento consiste em escolher, para cada posição do vetor do novo indivíduo, o valor da posição equivalente de um dos dois indivíduos selecionados, sendo tal decisão feita de forma aleatória. O mecanismo de cruzamento deve garantir que a soma dos tempos seja inferior a 120s. Portanto, caso este valor seja superado, deve-se decrementar $T_{\text {verde }}$ de cada uma das vias sequencialmente até o somatório se igualar a 120 .

O torneio e o cruzamento serão realizados até serem formados 100 novos indivíduos que irão compor a nova geração.

\subsubsection{Mutação de indivíduos}

A mutação é feita selecionando-se aleatoriamente alguns dos novos indivíduos, com base em uma porcentagem de $5 \%$ de chance (definido empiricamente), que terão uma de suas posições alterada (selecionada aleatoriamente) para um novo valor também aleatório que respeite o limite de 120 segundos para o ciclo. Tal procedimento é feito para prevenir que as soluções da população fiquem em um ponto ótimo local e para manter a diversidade.

Ao final de dez repetições dos procedimentos acima descritos, a melhor solução da população final é escolhida e apresentada. 


\subsection{Algoritmo genético para redes de cruza- mento}

Nesta seção, é descrita uma implementação do algoritmo genético para a otimização de redes de tráfego como um todo.

A técnica escolhida para o otimizador de redes de cruzamento busca combinar o algoritmo genético de cruzamentos isolados e um algoritmo genético para otimização apenas dos tempos de abertura iniciais. A ideia se baseia no fato de que será obtida uma boa configuração para cada cruzamento após a execução do otimizador individual. Assim, seria necessário apenas determinar bons tempos de atraso para abertura inicial de forma que o fluxo de veículos na rede seja melhor.

O algoritmo genético para a determinação dos tempos de abertura iniciais recebe como entrada uma configuração para os tempos $T_{\text {vermelho }}$ e $T_{\text {verde }}$ para todos os cruzamentos da rede. Deste modo, os indivíduos serão apenas um vetor de inteiros indicando o tempo de atraso para abertura inicial do cruzamento ao qual a posição do vetor se refere.

A seleção de indivíduos ocorre de forma semelhante ao torneio do algoritmo genético para cruzamentos isolados. A formação do novo indivíduo, entretanto, diferencia-se por ser a média dos tempos de abertura iniciais dos dois indivíduos pais, sendo uma média para cada cruzamento. A mutação tem $5 \%$ de chance de acontecer para cada indivíduo da população e consiste em alterar aleatoriamente o valor do tempo de abertura de um cruzamento também aleatório, respeitando-se o limite que o valor pode assumir.

O objetivo do algoritmo genético é minimizar o somatório dos tempos médios de espera de todas as vias de entrada da rede. Caso o usuário deseje dar prioridade à algumas vias, poderia ser feita uma soma ponderada, de forma que o algoritmo priorize a minimização do tempo de tais vias. Caso isso seja feito, deve-se multiplicar o peso pelo tempo médio de espera. Por ser mais simples, o número de indivíduos na população do algoritmo para determinação dos tempos iniciais é igual a 50 e o número de gerações é apenas 5 .

Um dos maiores problemas da abordagem que envolve combinar o algoritmo genético para cruzamentos isolados e o otimizador dos tempos de abertura iniciais é que só são conhecidos os tempos entre chegadas de veículos das vias fontes do sistema. Ou seja, para as vias de entrada internas ao cruzamento não é conhecido, inicialmente, o tempo médio entre chegadas de cada uma. O algoritmo desenvolvido para a otimização de redes de tráfego contorna este problema aplicando de forma alternada e sistemática o simulador de cruzamentos individuais, o algoritmo genético para otimização de cruzamentos individuais, o algoritmo genético para a otimização de tempos iniciais e o simulador de rede. Este algoritmo é descrito pelos seguintes passos:

1. Gera-se e simula-se uma configuração aleatória para a rede.

2. A partir da simulação, obtêm-se os tempos médios entre chegadas das vias internas da rede.

3. Executa-se, então, o algoritmo genético para cruzamentos individuais.

4. Utilizando a configuração final obtida pelo passo anterior, executa-se o algoritmo genético otimizador de tempos de abertura iniciais.
5. Simula-se o indivíduo obtido no passo anterior e retorne ao passo 2 .

Com base nos testes realizados, foi possível verificar que os passos anteriores devem ser executados três vezes, pois os melhores resultados são adquiridos até a terceira iteração. Números de repetições do processo além disso consomem mais processamento, tomam mais tempo e não costumam gerar soluções melhores.

\section{RESULTADOS}

Os algoritmos propostos neste trabalho foram implementados por meio da linguagem de programação Java, sem a utilização de nenhum framework adicional.

Para avaliar o simulador e otimizador de cruzamentos isolados, um caso de teste foi feito com base em um cruzamento real situado na cidade de Varginha, Minas Gerais. O cruzamento em questão é composto por duas vias de entrada (cada uma com apenas uma faixa) e duas de saída. Uma gravação de vídeo foi realizada por 20 minutos nesse cruzamento para possibilitar a coleta de dados que seriam necessários para a execução do simulador.

A partir do estudo realizado com base na gravação da primeira via de entrada foi possível identificar que a média de tempo entre chegadas de veículos era de 8,8s. Durante o período analisado, 134 veículos passaram pelo cruzamento a partir dessa via, sendo que o tempo médio de espera destes veículos foi de 13,09 s. Já em relação à segunda via de entrada, 66 carros passaram por tal via, com tempo médio entre chegadas de 18,5s. O tempo médio de espera para essa via foi de 17,56s. Foi identificado que o semáforo da primeira via ficava aberto por $33 \mathrm{~s}$ e fechado por $22 \mathrm{~s}$. O oposto era válido para a segunda via, que ficava verde por 22 s e vermelho por 33s. Além disso, foi verificado que o tempo médio de reação à abertura do semáforo no cruzamento foi de $4,1 \mathrm{~s}$ e que o tempo médio para travessia foi de $3,4 \mathrm{~s}$. Um diagrama ilustrando este caso de teste é apresentado na Figura 1. Na figura, o cruzamento é representada por um nó, enquanto as vias são representadas por setas orientadas. Ao lado de cada via de entrada é exibido o rótulo V.E., seguido do número da via, a média de tempo de chegada entre veículos e o tempo em que o sinal fica aberto.

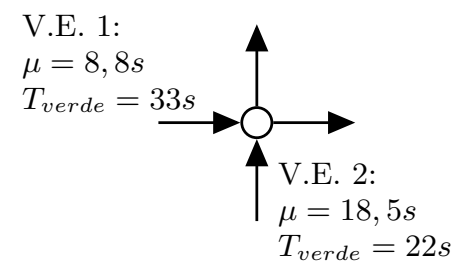

\section{Figura 1: Caso 1 - Cruzamento em Varginha-MG}

Com base nos dados anteriores, a simulação foi executada para verificar a precisão dos resultados em relação à realidade e o otimizador foi executado para identificar possibilidades de melhoria nos tempos semafóricos. As informações geradas se encontram na Tabela 1. A coluna Realidade apresenta as informações do caso real, a coluna Simulação apresenta os resultados obtidos pelo simulador, utilizando os parâmetros do caso real, a coluna Otimização indica os resultados obtidos ao se simular a solução encontrada pelo otimizador. 
Tabela 1: Simulação caso 1

\begin{tabular}{|c|c|c|c|c|c|c|}
\hline \multirow[b]{2}{*}{ Via de entrada } & \multicolumn{2}{|c|}{ Realidade } & \multicolumn{2}{|c|}{ Simulação } & \multicolumn{2}{|c|}{ Otimização } \\
\hline & 1 & 2 & 1 & 2 & 1 & 2 \\
\hline T. verde (s) & 33 & 22 & 33 & 22 & 21 & 11 \\
\hline T. vermelho (s) & 22 & 33 & 22 & 33 & 11 & 21 \\
\hline Num. veículos & 134 & 66 & 9923 & 4675 & 9770 & 4588 \\
\hline T. médio de espera (s) & 13,09 & 17,56 & 13,65 & 16,64 & 11,01 & 15,4 \\
\hline T. simulado ou gravado & 20 minutos & 20 minutos & 24 horas & 24 horas & 24 horas & 24 horas \\
\hline
\end{tabular}

Verifica-se que o simulador, utilizando os mesmos tempos de semáforo da gravação, produz valores bem próximos aos reais. Além disso, o otimizador recomendou uma mudança que poderia resultar em tempos médios de espera menores para ambas as vias de entrada. Apesar dos resultados promissores, é importante ressaltar que os dados coletados na gravação compreenderam um tempo curto de um único dia. Portanto, seria necessário uma análise mais profunda, compreendendo vários dias e períodos dos dias, para uma validação mais completa do simulador.

Para avaliar o simulador e o otimizador de redes de tráfego, foi gerado um caso de teste hipotético. A rede testada é apresentada na Figura 2. Os cruzamentos são representados por nós e as vias por setas. Os nós preenchidos com cor cinza representam cruzamentos não sinalizados, os demais representam cruzamentos sinalizados. As setas sem nó de origem representam origem de veículos para o sistema, os rótulos próximos à estas setas representam os tempos médios, $\mu$, entre chegadas de veículos. As setas sem nó de destino representam a saída de veículos do sistema. Os rótulos apresentados próximos às setas conectando dois nós representam o tempo médio para se deslocar entre os respectivos cruzamentos. O tempo médio de reação à abertura do sinal e o tempo médio entre saídas de veículos consecutivos foram considerados os mesmos que foram obtidos pela gravação em vídeo do cruzamento utilizado no otimizador de cruzamentos individuais, ou seja, 4,1s e 3,4s, respectivamente. Os resultados obtidos pelo otimizador encontram-se nas tabelas 2 e 3 . A linha Via de cada tabela indica a via para as quais as informações são exibidas, no formato (origem,destino). As vias são apresentadas para cada cruzamento em ordem de abertura dos respectivos semáforos. Para a obtenção dos resultados, os simuladores são executados por um tempo equivalente à 24 horas. É importante notar que as vias de cruzamentos não sinalizados que possuem tempo médio de espera igual a zero indica que os movimentos realizados em tais vias são sempre prioritários, ou seja, não precisam esperar para serem realizados.

De acordo com o simulador, a solução encontrada pelo otimizador leva a um tempo de espera médio em cada via de aproximadamente 12,14s. Em contraste, ao executarmos o simulador com um tempo de sinal verde igual a 30 s para cada via, obtemos um tempo médio de espera em cada via de aproximadamente $730,85 \mathrm{~s}$.

\section{CONCLUSÕES}

Neste trabalho, foram desenvolvidos algoritmos de simulação e otimização para sistemas de sinalização semafórica, em particular, cruzamentos isolados e redes de tráfego. Os algoritmos de simulação se baseiam no paradigma de simulação orientada a eventos discretos, enquanto os algoritmos de otimização se baseiam no algoritmo genético.

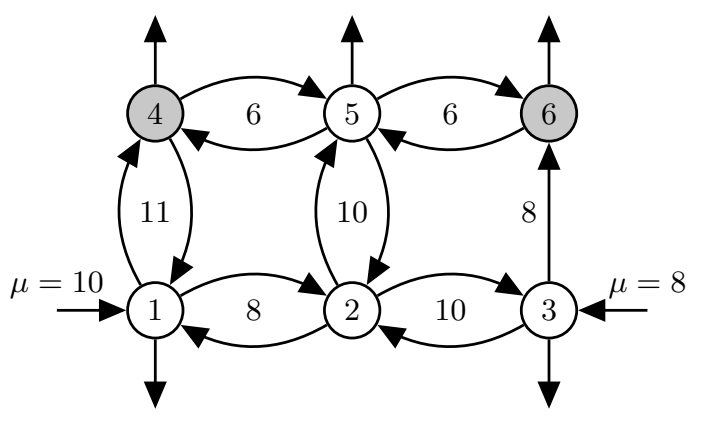

Figura 2: Caso 2 - Teste hipotético.

Os algoritmos foram testados em um caso de teste real e um artificial, apresentando bons resultados. Acreditamos que os algoritmos desenvolvidos neste trabalho podem ser ferramentas úteis de apoio à tomada de decisões no momento da definição das configurações semafóricas, seja para cruzamentos isolados ou redes de cruzamentos.

Existem várias melhorias que podem ser feitas no programa desenvolvido, sejam elas adições de funcionalidades ainda não existentes ou evoluções das já implementadas. Tais melhorias servem como possibilidades para trabalhos futuros. Entre elas, podem-se citar:

- Considerar que uma via pode se sobrecarregar, impedindo que outros veículos sigam naquela direção enquanto estiver cheia.

- Incluir a passagem de pedestres como um fator a ser considerado no momento da otimização, buscando diminuir o tempo de espera dos mesmos para a travessia.

- Permitir que a decisão de formação de grupos semafóricas seja também parte do algoritmo otimizador, e não determinada somente pelo usuário.

- Desenvolver uma interface gráfica intuitiva para facilitar o uso do software.

- Paralelizar o algoritmo para execuções em menor tempo.

O código fonte deste trabalho encontra-se disponível sob uma licença livre através do endereço https://github.com/ matheusbarrosp/SimuladorOtimizadorSemaforico. Dessa maneira, é possível que mais testes sejam realizados e que sejam implementadas as melhorias, não só pelos desenvolvedores do software, como também por qualquer um que tenha interesse.

Os algoritmos ainda precisam passar por mais testes e, possivelmente, avaliações por parte de especialistas de trânsito, para que sejam completamente validados. Entretanto os resultados iniciais demonstram o potencial da ferramenta 
Tabela 2: Otimização - caso 2

\begin{tabular}{|c|c|c|c|c|c|c|c|c|}
\hline \multirow[b]{2}{*}{ Via } & \multicolumn{3}{|c|}{ Cruzamento 1} & \multicolumn{3}{|c|}{ Cruzamento 2} & \multicolumn{2}{|c|}{ Cruzamento 3} \\
\hline & $(4,1)$ & $(2,1)$ & $(-, 1)$ & $(5,2)$ & $(3,2)$ & $(1,2)$ & $(-, 3)$ & $(2,3)$ \\
\hline T. verde $(\mathrm{s})$ & 11 & 16 & 29 & 11 & 15 & 15 & 22 & 11 \\
\hline T. primeira abertura (s) & & 21 & & & 19 & & & \\
\hline Num. veículos & 855 & 3447 & 8530 & 1559 & 5314 & 4495 & 10694 & 2969 \\
\hline T. médio de espera (s) & 24,56 & 22,99 & 17,09 & 15,47 & 18,15 & 15,93 & 9,91 & 12,67 \\
\hline
\end{tabular}

Tabela 3: Otimização - caso 2 (continuação)

\begin{tabular}{|c|c|c|c|c|c|c|c|}
\hline \multirow[b]{2}{*}{ Via } & \multicolumn{2}{|c|}{ Cruzamento 4} & \multicolumn{3}{|c|}{ Cruzamento 5} & \multicolumn{2}{|c|}{ Cruzamento 6} \\
\hline & $(5,4)$ & $(1,4)$ & $(6,5)$ & $(2,5)$ & $(4,5)$ & $(3,6)$ & $(5,6)$ \\
\hline T. verde $(\mathrm{s})$ & - & - & 18 & 21 & 11 & - & - \\
\hline T. primeira abertura (s) & \multicolumn{2}{|c|}{-} & \multicolumn{3}{|c|}{15} & \multicolumn{2}{|c|}{-} \\
\hline Num. veículos & 2153 & 4943 & 3755 & 4952 & 2470 & 7474 & 1892 \\
\hline T. médio de espera (s) & 3,20 & 0 & 15,47 & 15,62 & 23,41 & 1,79 & 0 \\
\hline
\end{tabular}

em gerar bons resultados, como uma simulação fiel à realidade e diminuição do tempo de espera por meio da otimização.

\section{REFERÊNCIAS}

[1] E. Angulo, F. P. Romero, R. García, J. Serrano-Guerrero, and J. A. Olivas. A Methodology for the Automatic Regulation of Intersections in Real Time Using Soft-Computing Techniques, pages 379-388. Berlin, Heidelberg, 2008.

[2] A. Barisone, D. Giglio, R. Minciardi, and R. Poggi. A macroscopic traffic model for real-time optimization of signalized urban areas. In Proceedings of the 41st IEEE Conference on Decision and Control, 2002., volume 1, pages 900-903 vol.1, Dec 2002.

[3] S. G. de Souza Cervantes, J. C. Piai, E. F. F. Ramírez, L. Varasquim, and E. Nagayama. Atefi-um algoritmo para controle semafórico em tempo fixo descentralizado. Semina: Ciências Exatas e Tecnológicas, 30(1):41-50, 2009.

[4] M. B. d. S. de Trânsito. Volume v. Sinalização semafórica, 2007.

[5] R. d. S. dos Santos, W. A. Paquerote, and W. K. da Silva Soares. Simulação de trânsito e semáforos: Um estudo de caso piloto em um trecho no entorno do campus da ufrn. In ANAIS DO XLVII SBPO, 2015.

[6] M. Dotoli, M. P. Fanti, and C. Meloni. Coordination and real time optimization of signal timing plans for urban traffic control. In IEEE International Conference on Networking, Sensing and Control, 2004, volume 2, pages 1069-1074, 2004.

[7] D. E. Golberg. Genetic Algorithms in Search, Optimization, and Machine Learning. Addison-Wesley, 1989.

[8] F. S. Hillier. Introduction to operations research. McGraw-Hill, 2006.

[9] L. M. Leemis and S. K. Park. Discrete-event simulation: A first course. Pearson Prentice Hall Upper Saddle River, NJ, 2006.

[10] L. C. F. Lopes. MÉTODO DE OTIMIZAÇÃO DAS DEFASAGENS DE CORREDORES ARTERIAIS. PhD thesis, Universidade Federal do Rio de Janeiro, 2010.
[11] M. Papageorgiou, C. Diakaki, V. Dinopoulou, A. Kotsialos, and Y. Wang. Review of road traffic control strategies. Proceedings of the IEEE, 91(12):2043-2067, Dec 2003. 